\title{
Acoustic Scattering Performance of a Duct for a Monopole Source Using Thin-Body BEM Method
}

\author{
Chuxiong Yan ${ }^{a}$, Jun Huang ${ }^{b}$, Mingxu $\mathrm{Yi}^{\mathrm{c}}$, Yacong $\mathrm{Wu}{ }^{\mathrm{d}}$
}

School of Aeronautic Science and Engineering, Beihang University, Beijing 100191, China

ayanchuxiong4@163.com, bjunhuang1964@163.com, cyimingxu88@163.com, dwycbuaa@163.com

\begin{abstract}
In this paper, in order to calculate the acoustic scattering of the duct, a thin-body boundary element method (BEM) has been proposed and the velocity obtained by the acoustic velocity formulation is use1d as the Neumann boundary condition on a rigid scattering surface. The radiated sound pressure and the scattering effect of the solid wall on the propagation of the sound wave are calculated by using the Kirchhoff formulation and the thin-body BEM, respectively. Computational results for a monopole source verify the method. The sound pressure directivity and scattering effect are shown to demonstrate the applicability and validity of the approach.
\end{abstract}

Keywords: Acoustic Scattering Effect; Boundary Element Method; Velocity Formulation.

\section{Introduction}

Noise pollution has become an annoying problem in recent years and a great deal of progress has been made to predict the noise generated by rotating blade. In these applications, in order to evaluate the sound pressure for any observer location, the incident sound field and the scattering sound field should be considered.

Scattering bodies located near sources can convert some of their intense energy near field into the form of sound waves whose amplitudes are far greater than that of the incident field [1]. Several numerical and analytical methods have been applied to predict the acoustic scattering in turbo machinery, aircraft engines and the rotor noise by the airframe.

The acoustic Boundary Element Method has been applied to solve acoustic radiation and scattering problem in the exterior and interior closed domain for long time [2, 4]. However, the traditional BEM has difficulty in solving the problem of thin body as the meshes on two sides are quite close. Thus, by constructing an imaginary surface in the acoustic domain, researchers divide it into interior and exterior part and then the Helmholtz Integral Equation [5, 6] can be applied.

This method need to calculate of acoustic velocity or the acoustic pressure gradient on the scattering surface to meet the boundary condition. A direct numerical evaluation of the pressure gradient can be expensive for realistic cases. Ghorbaniasl et al. [7] obtained an acoustic velocity formulation based on the Kirchhoff formulation, which could be used as the boundary condition in this method and then improve the computational efficiency.

The layout of this work is as follows: firstly, the total sound pressure is divided into the incident and scattered pressure. Secondly, the thin-body BEM is built up and the incident and scattered pressure are calculated respectively. Thirdly, acoustic scattering performance of a duct for a monopole source is studied and some useful conclusions have been made.

\section{Thin-body Acoustic Boundary Element Method}

The thin-body acoustic BEM is a way to compute the far field acoustic pressure. The total sound pressure in the far field can be computed by summing the incident and scattered pressure.

$P^{\prime}(x, \omega)=P_{I}^{\prime}(x, \omega)+P_{S}^{\prime}(x, \omega)$

Where $P^{\prime}$ is the total sound pressure in the frequency domain, and the incident pressure $P_{I}^{\prime}$ and the scattered pressure $P_{S}^{\prime}$ are also in the frequency domain. The incident sound pressure can be calculated 
by using Eq. (2) and transformed into frequency dominant data by executing Fast Fourier Transform [8].

$$
p(\vec{x}, t)=\frac{1}{4 \pi} \int_{s}\left[\frac{E_{1}}{r\left(1-M_{r}\right)}+\frac{E_{2}}{r^{2}\left(1-M_{r}\right)}\right]_{r e t} d S
$$

$E_{1}$ and $E_{2}$ are given as:

$$
\begin{aligned}
E_{1}= & M_{n} M_{t} \cdot \nabla_{2} p^{\prime}-\frac{M_{n} p^{\prime}}{c_{0}}+\left(M_{n}^{2}-1\right) p_{n}^{\prime}+\frac{\left(n_{r}-M_{n}-n_{M}\right) p^{\prime}+\left(n_{r}-M_{n}\right) p^{\prime}}{c_{0}\left(1-M_{r}\right)} \\
& +\frac{M_{r}\left(n_{r}-M_{n}\right) p^{\prime}}{c_{0}\left(1-M_{r}\right)^{2}} \\
E_{2}= & \frac{1-M^{2}}{(1-M)^{2}}\left(n_{r}-M_{n}\right)
\end{aligned}
$$

The scattered sound pressure can be solved by using BEM. The calculated domain is shown in the Fig.1, the surface of the duct is represented as $S$ and the imaginary $s$ is assumed to make the duct closed and divide the whole domain into two parts: the interior subdomain $D^{+}$and an exterior subdomain $D^{-}$. The sound pressure outside the surface $S+s$ is denoted by $P^{\prime-}$ and that on the inside is denoted by $P^{\prime+}$. The integral equation can be used to each subdomain [9].

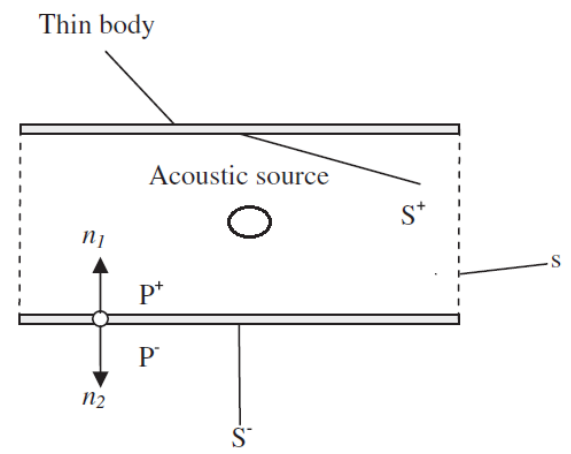

Fig. 1 A diagram of acoustic scattering by a thin-body.

The thin-body boundary integral equation can be obtained:

$$
\begin{aligned}
& \frac{1}{2}\left[P^{\prime+}(x, \omega)+P^{\prime-}(x, \omega)\right]=P_{I}^{\prime}(x, \omega)+\int_{S}\left\{G(x, y, \omega)\left[\frac{\partial P^{\prime+}(y, \omega)}{\partial n(y)}-\frac{\partial P^{\prime-}(y, \omega)}{\partial n(y)}\right]\right. \\
&\left.-\frac{\partial G(x, y, \omega)}{\partial n(y)}\left[P^{\prime+}(y, \omega)-P^{\prime-}(y, \omega)\right]\right\} d S(y), x \in S \\
& P^{\prime}(x, \omega)= P_{I}^{\prime}(x, \omega)+\int_{S}\left\{G(x, y, \omega)\left[\frac{\partial P^{\prime+}(y, \omega)}{\partial n(y)}-\frac{\partial P^{\prime-}(y, \omega)}{\partial n(y)}\right]\right. \\
&\left.-\frac{\partial G(x, y, \omega)}{\partial n(y)}\left[P^{\prime+}(y, \omega)-P^{\prime-}(y, \omega)\right]\right\} d S(y), x \in D^{+} \cup D^{-} \cup s
\end{aligned}
$$

Where $\partial / \partial n_{1}=-\partial / \partial n_{2}=\partial / \partial n$, and since the continuous boundary conditions of the pressure and its partial derivation on the imaginary surface $s$ are used, one obtains the following relation

$$
P^{\prime^{+}}(x, \omega)={P^{\prime}}^{-}(x, \omega), \quad \frac{\partial{P^{\prime+}}^{\prime}(x, \omega)}{\partial n(x)}=\frac{\partial{P^{\prime}}^{-}(x, \omega)}{\partial n(x)}, \quad x \in s
$$

The assumption of acoustic rigid boundary conditions is applied over the entire surface $S$

$$
\frac{\partial P^{\prime+}(x, \omega)}{\partial n(x)}=\frac{\partial P^{\prime-}(x, \omega)}{\partial n(x)}=0, \quad x \in S
$$

Then Eq. (4) and Eq. (5) can be simplified to 


$$
\begin{gathered}
\frac{1}{2}\left[P^{\prime+}(x, \omega)+P^{\prime-}(x, \omega)\right]=P_{I}^{\prime}(x, \omega) \\
\quad-\int_{S}\left\{\frac{\partial G(x, y, \omega)}{\partial n(y)}\left[P^{\prime+}(y, \omega)-P^{\prime-}(y, \omega)\right]\right\} d S(y), x \in S \\
P^{\prime}(x, \omega)=P_{I}^{\prime}(x, \omega)-\int_{S}\left\{\frac{\partial G(x, y, \omega)}{\partial n(y)}\left[P^{\prime+}(y, \omega)-P^{\prime-}(y, \omega)\right]\right\} d S(y), x \in D^{+} \cup D^{-} \cup s
\end{gathered}
$$

The two unknowns $P^{\prime+}(x, \omega)$ and $P^{\prime-}(x, \omega)$ are not obtained only by solving the Eq. (9). Differentiating Eq. (8) with regard to the direction of normal vector $n(x)$, it can be transformed into

$$
0=\frac{\partial P_{I}^{\prime}(x, \omega)}{\partial n(x)}-\int_{S}\left\{\frac{\partial^{2} G(x, y, \omega)}{\partial n(y) \partial n(x)}\left[P^{\prime+}(y, \omega)-P^{\prime-}(y, \omega)\right]\right\} d S(y), x \in S
$$

In order to calculate the acoustic pressure at any filed point, Eq.(10) is solved to get the sound pressure jump $P^{\prime+}(y, \omega)-P^{\prime-}(y, \omega)$ on the surface $S$ and substituted into Eq.(9). Then the acoustic pressure on both sides of the duct could be easily obtained. However, the value of $\frac{\partial P_{I}^{\prime}(x, \omega)}{\partial n(x)}$ cannot be obtained easily by using Eq. (2). We can acquire it indirectly by using the acoustic velocity formulation. If Eq. (11) satisfies the Neumann boundary condition, one has

$$
\frac{\partial P_{I}^{\prime}(x, \omega)}{\partial n(x)}=-i \omega \rho_{0} v_{n}(x, \omega)
$$

Where $v_{n}=\left\{a_{1}^{\prime}(x, \omega), a_{2}^{\prime}(x, \omega), a_{3}^{\prime}(x, \omega)\right\}$ for the acoustic sources and $a_{i}$ could be get by the following equation:

$$
\begin{aligned}
4 \pi \rho_{0} a_{i}^{\prime}(x, t) & =\frac{1}{c_{0}} \int_{S}\left[I_{k} \hat{r}_{i}\right]_{e} d s \\
& -\frac{1}{c_{0}} \int_{S}\left[\frac{p^{\prime}\left(\cos \theta-M_{n}\right)\left(M_{i}-\hat{r}_{i}\right)}{r^{2}\left(1-M_{r}\right)^{2}}+\frac{p^{\prime}\left(\hat{n}_{i}-\cos \theta \hat{r}_{i}\right)}{r^{2}\left(1-M_{r}\right)}\right]_{e} d s \\
& \left.-\int_{0}^{t}\left\{\int_{S}\left[\frac{p^{\prime}\left(\hat{n}_{i}-3 \cos \theta \hat{r}_{i}\right)}{r^{3}\left(1-M_{r}\right)}\right]_{e^{*}}+\left[\frac{\left(p_{n}^{\prime}+\frac{M_{n}}{c_{0}} p_{t}^{\prime}\right) \hat{r}_{i}}{r^{2}\left(1-M_{r}\right)}\right]_{e^{*}}\right] d s\right\} \quad i=1,2,3
\end{aligned}
$$

\section{Numerical results}

In this test case, the comparison of the analytical solution and the numerical algorithm of a 3-D monopole source is used to verify the algorithm. The monopole is identified with a pulsating sphere as the small sphere with a radius. The pressure fluctuation induced by the pulsating sphere is expressed by a harmonic spherical wave

$$
p^{\prime}=\frac{A \omega \rho_{0}}{4 \pi r} \frac{1}{\sqrt{1+(k a)^{2}}} \cos \left[\omega t-k(r-a)+\phi_{0}\right]
$$

Where $\omega_{\text {and }} k$ are angular velocity and the wave number, respectively, and

$$
\phi_{0}=\tan ^{-1}\left(\frac{1}{k a}\right), \quad r=\sqrt{x^{2}+y^{2}+z^{2}}
$$

The velocity is given by 


$$
\begin{aligned}
u_{r}(r, t) & =\frac{1}{\rho_{0}} \int \frac{\partial p^{\prime}}{\partial r} d t \\
& =\frac{1}{\sqrt{1+(k a)^{2}}}\left(\frac{A k}{4 \pi r} \cos \left[\omega t-k(r-a)+\varphi_{0}\right]+\frac{A}{4 \pi r^{2}} \sin \left[\omega t-k(r-a)+\varphi_{0}\right]\right)
\end{aligned}
$$

Where $A=4 \pi a^{2} U$. To perform this case, the radius of the spherical penetrable data surface $r_{s}$ is assumed as $3.25 a$. The speed of sound $c_{0}$ is $340 \mathrm{~m} / \mathrm{s}$. The density for medium is $1.2 \mathrm{~kg} / \mathrm{m} 3$. The angular velocity of the source is $340 \mathrm{rad} / \mathrm{s}$. The other parameters are $a=0.01 \mathrm{~m}$ and $U=8 \mathrm{~m} / \mathrm{s}$. The pulsating sphere is located at the center of the duct. The diameter of the duct is $0.07 \mathrm{~m}$. The length of the duct is $0.5 \mathrm{~m}$. The observer distance is assumed to be $1 \mathrm{~m}$.

The $x, y$ and $z$ components of the acoustic velocity obtained with the velocity formulation are calculated by using the numerical method. The results are compared with analytical solutions for different observer time which are plotted in Figs.2. From Figs.2, we find that the numerical solutions are in very good agreement with the analytical solution, which could be used in the next procedure. To perform the acoustic scattering problems of the duct, we use the thin-body BEM. Fig.3 shows the scattering performance of the pulsating sphere. The left is the incident sound pressure, the right is the scattering effect of the duct and the middle is the total sound pressure. The sound pressure is expressed as $\mathrm{dB}$ (decibels) and the predicted SPLs (sound pressure levels) is given by the following

$$
S P L s=20 \lg \frac{P_{e}}{P_{r}}
$$

Where is predicted pressure, denotes the reference pressure and equals to $2 \times 10-5 \mathrm{~Pa}$.
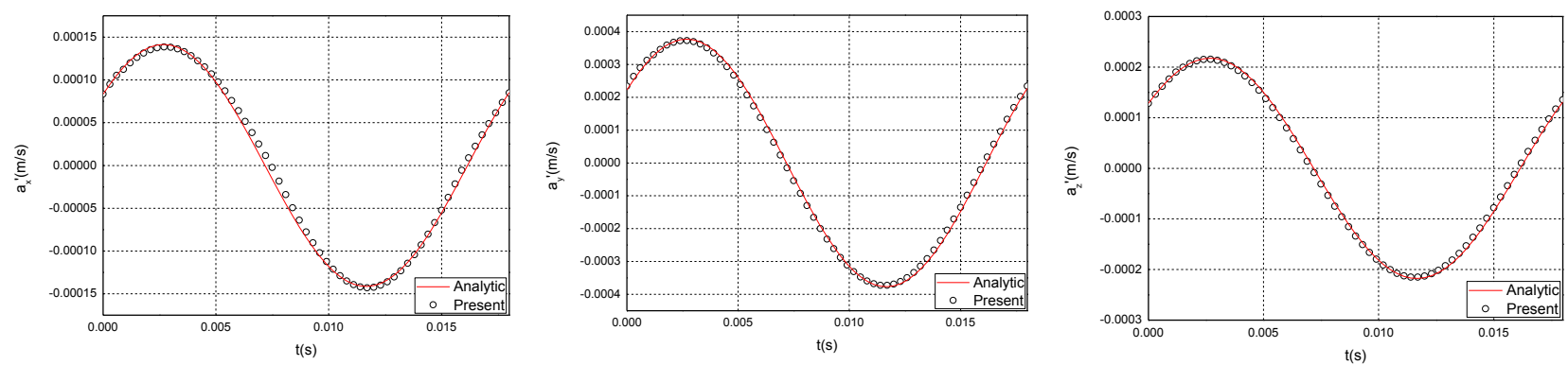

Fig.2 The calculated $x, y$ and $z$ component of acoustic velocity compared with that of the analytical solution.
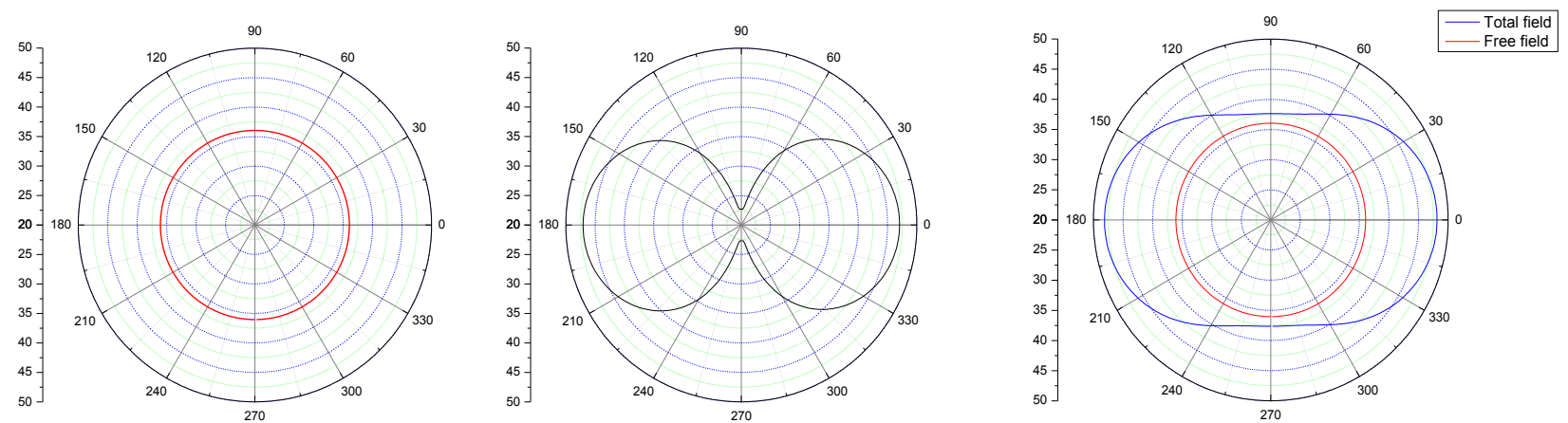

Fig.3 Directivity of calculated far-field SPLs at $4918 \mathrm{~Hz}$ (a) free field, (b) scattering effect of the duct and (c) total field.

The Fig. 3 shows the directivity of the incident sound pressure is circular as the property of monopole sound source. When the scattering effect is considered, the directivity of the sum sound becomes non-circular but still symmetrical. For the angle (-45 to 45 degrees and 135 to 235 degrees), SPLs of the total field is louder due to scattering where at the angle (45to 135 degrees and 235 to 315 degrees), it is slightly louder. It shows that the sound pressure is strengthened largely in the direction of duct both ends and that in the direction of the wall is relatively small. 


\section{Conclusion}

This paper develops the thin-body BEM method for calculating the scattering effect of a duct numerically. The acoustic velocity formulation can be utilized as boundary condition for thin-body BEM. Furthermore, a verification study is given. For the monopole source, due to the scattering effect, the amplitude of SPLs is greater and directivity is changed from circular to symmetrical.

\section{References}

[1]. Crighton D G, Leppington F G. On the scattering of aerodynamic noise. Journal of Fluid Mechanics. Vol. 46 (1971) No. 3, p. 577-597.

[2]. Wu H, Liu Y, Jiang W. A low-frequency fast multipole boundary element method based on analytical integration of the hypersingular integral for 3D acoustic problems. Engineering analysis with boundary elements. Vol. 37 (2013) No. 2, p. 309-318.

[3]. Peake M J, Trevelyan J, Coates G. Extended isogeometric boundary element method (XIBEM) for two-dimensional Helmholtz problems. Computer Methods in Applied Mechanics and Engineering. Vol. 259 (2013), p. 93-102.

[4]. Zheng C, Matsumoto T, Takahashi T, et al. A wideband fast multipole boundary element method for three dimensional acoustic shape sensitivity analysis based on direct differentiation method. Engineering Analysis with Boundary Elements. Vol. 36 (2012) No. 3, p. 361-371.

[5]. Mao Y, Qi D, Liu X, et al. Numerical prediction of aerodynamic tonal noise radiated from a centrifugal fan. Proceedings of the Institution of Mechanical Engineers, Part A: Journal of Power and Energy. Vol. 222 (2008) No. 8, p. 831-842.

[6]. Zhu J, Shah A H, Datta S K. The evaluations of Cauchy Principal Value integrals and weakly singular integrals in BEM and their applications. International journal for numerical methods in engineering. Vol. 39 (1996) No. 6, p. 1017-1028.

[7]. Ghorbaniasl G, Hirsch C, Siozos-Rousoulis L, et al. Acoustic velocity formulation for Kirchhoff data surfaces. International Journal of Aeroacoustics. Vol. 14 (2015) No. 1-2, p. 105-132.

[8]. Farassat F, Myers M K. Extension of Kirchhoff's formula to radiation from moving surfaces [J]. Journal of Sound and Vibration. Vol. 123 (1988) No. 3, p. 451-460.

[9]. Mao Y, Qi D. Computation of rotating blade noise scattered by a centrifugal volute. Proceedings of the Institution of Mechanical Engineers, Part A: Journal of Power and Energy. Vol. 223 (2009) No. 8, p. 965-972. 